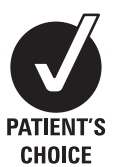

CHOICE

\title{
Cognitive behavioural group therapy to improve patients' strategies for coping with restless legs syndrome: a proof-of-concept trial
}

\author{
M Hornyak, ${ }^{1}{ }^{12}$ C Grossmann, ${ }^{1}$ R Kohnen, ${ }^{3}$ M Schlatterer, ${ }^{1}$ H Richter, ${ }^{1}$ U Voderholzer, ${ }^{1}$ \\ D Riemann, ${ }^{1}$ M Berger ${ }^{1}$
}

\begin{abstract}
- An addendum is published online only at http://jnnp.bmj. com/content/vol79/issue7

${ }^{1}$ Department of Psychiatry and Psychotherapy, University Medical Centre, Freiburg, Germany; ${ }^{2}$ Interdisciplinary Pain Centre, University Medical Centre, Freiburg, Germany; ${ }^{3}$ IMEREM, Nuremberg, and Department of Psychology, University Erlangen-Nuremberg, Germany
\end{abstract}

Correspondence to:

Dr M Hornyak, Interdisciplinary Pain Centre and Department of Psychiatry and Psychotherapy, University Medical Centre, Breisacher Strasse 64, D-79106 Freiburg, Germany; magdolna. hornyak@uniklinik-freiburg.de

Received 5 November 2007 Revised 1 February 2008 Accepted 7 February 2008 Published Online First 26 February 2008

\begin{abstract}
Background: Restless legs syndrome (RLS) is a usually chronic disorder accompanied by clinically relevant psychosocial impairment. To date, no psychologically based approach is available to improve the coping strategies and quality of life of RLS sufferers.

Objective: To develop cognitive behavioural therapy tailored to this disorder (the RELEGS coping therapy programme) and present the results of this proof-ofconcept study.
\end{abstract}

Methods: Twenty-five patients (five men, 20 women; 15 medicated, 10 unmedicated; mean (SD) age 56.1 (12.3) years) with subjective psychosocial impairment due to RLS participated in one of three consecutive therapy groups. The severity scales (IRLS and RLS-6) indicated moderate to severe RLS symptoms at baseline. Exclusion criteria were secondary RLS, foreseeable change of RLS medication during the study period, serious physical or psychiatric comorbidity, and severe cognitive deficits. Each group took part in eight group sessions (90 min each with a break).

Results: At the end of the treatment, both the RLSrelated quality of life and the mental health status of the subjects had improved significantly (QoL-RLS scale: from 28.6 (12.8) to 23.4 (13.1); SCL-90-R: from 51.3 (37.0) to 45.9 (32.9)). The improvement remained at follow-up 3 months later. Subjective ratings of RLS severity had improved at the end of therapy and at follow-up. Psychometric scales not specific for RLS-related impairment remained unaffected by the treatment.

Conclusions: The study establishes the feasibility and high acceptance of the newly devised therapy programme. The application of RLS-oriented specific psychological strategies is a step toward an integrated treatment approach in RLS.

Restless legs syndrome (RLS), a common sensorimotor disorder, ${ }^{1}$ usually has a chronic course with progression of symptom intensity in older people. ${ }^{2}$ In clinical trials, dopaminergic substances showed good efficacy in the short-term treatment of RLS, although complete remission of symptoms does not occur in all patients, ${ }^{3}$ and long-term treatment with dopaminergic drugs may be complicated by loss of efficacy and augmentation. ${ }^{4}$ Symptom persistence has an effect on the general condition of patients, and recent studies have shown a high incidence of psychosocial impairment, ${ }^{5-7}$ a reduced quality of life, ${ }^{1}$ and an increased prevalence of depressive and anxiety disorders associated with RLS. $^{8}$
We developed a psychologically based group therapy approach tailored to the specific aspects of the disorder, with the aim of improving coping strategies and quality of life of patients with RLS (the RELEGS, Restless Legs Skills programme). The programme integrates cognitive behavioural elements and acceptance-based mindfulness approaches $^{910}$ (for the conception of the study, see the addendum published online as supplementary material). The presented study is a proof-ofconcept investigation to evaluate the feasibility, patient acceptance and efficacy of the group therapy.

\section{PATIENTS AND METHODS}

Approval for the study was granted by the local ethics committee. All patients gave their written informed consent before participating in the study.

\section{Design}

For this pilot study, we performed a pre-post comparison of outcome measures taken at baseline, at an intermediate mid-treatment assessment after 4 weeks, and at the final visit after conclusion of the group therapy as well as at follow-up. There was no control group. Evaluations of outcome parameters were performed by an independent rater who was not involved in any of the therapy procedures.

\section{Patients}

The group therapy was offered to a cohort of all patients with RLS seen during the study period at our Sleep Disorders Outpatient Unit. RLS diagnosis was based on accepted criteria. ${ }^{2}$ For inclusion, patients must have reported in the clinical interview bothersome psychosocial impairment due to RLS. Both medicated and unmedicated patients were included in the study. Some of the medicated patients did not wish a further increase in dose. In other cases, a further increase in dose or a change of dopamine agonists or add-on therapies (eg, opiates) led to barely tolerable side effects. Exclusion criteria were secondary RLS (due to an underlying disorder known to trigger RLS, eg, renal failure, autoimmune disorders) serious physical comorbidity with possible deterioration of quality of life (eg, neurodegenerative disorders, active malignant tumours), serious psychiatric comorbidity (eg, severe depression with suicidality, post-traumatic stress disorder, substance dependency) and severe cognitive deficits. The screening procedure 
consisted of a semi-structured interview to ascertain history of sleep disturbance, physical examinations, serum biochemistry and the Structured Clinical Interview for DSM-IV-TR. ${ }^{11}$ Patients were untreated or could continue their current RLS medication if treatment response had been stable for 4 weeks before screening and no change of RLS medication during the study period was foreseeable.

\section{Group setting}

Three consecutive groups underwent a structured therapy programme. Each group session was chaired by two therapists, both trained in sleep medicine and psychotherapy. The therapists worked in the Sleep Disorders Outpatient Unit and were familiar with the clinical picture of RLS. After training of both therapists in the modules of the RELEGS programme, group sessions for each group took place on a weekly basis for a total of eight sessions of 90 min each (each session with a break).

\section{Contents of the group therapy, questionnaires and psychometric scales}

For a detailed description, see the addendum published online.

\section{Statistical analysis}

To test for treatment effects, statistical analysis of the withinsubject changes used the Wilcoxon signed rank test. $p$ Values (two-sided) $<0.05$ were considered indicative of non-random changes, and all test results were interpreted in an exploratory manner. The primary outcome measure was the change in the RLS-specific quality of life (QoL-RLS) ${ }^{12}$ total score. The analyses were based on data from all participants; two patients without valid values at the follow-up assessment were excluded from analysis (one patient took additional RLS-specific medication during the follow-up period, and the other patient forgot to fill in the SF-36 questionnaire ${ }^{13}$ at follow-up). Unmedicated patients and patients receiving RLS-specific medication were compared using the Mann-Whitney test. For this study, three coping therapy groups were planned with at least seven participants per group to evaluate stability of effects.

\section{RESULTS}

\section{Participants}

Data from 25 patients (five men, 20 women) in three groups were evaluated (nine, seven and nine participants in the groups, respectively). All patients completed the coping therapy programme. Most patients were middle-aged (mean (SD) 56.1 (12.3) years; range 35-76). In the Structured Clinical Interview for DSM-IV-TR, two patients were diagnosed as having a moderate recurrent depressive episode without suicidality, and two others had generalised anxiety disorder. The remaining patients had no psychiatric comorbidity. Ten patients were unmedicated, and 15 patients continued with their previous RLS-specific medication, which had not been changed during the study period (L-dopa, pramipexole, ropinirole, cabergoline and combinations of these). Unmedicated and medicated patients did not differ in statistical terms with regard to their age and their baseline scores (see the addendum, table 1A). The severity scales (IRLS, RLS-6) indicated moderate to severe RLS symptoms (table 1)

\section{Psychosocial scales}

Table 1 summarises the changes in the evaluated scales. There was a remarkable improvement at the end of the coping therapy course in RLS-specific aspects of quality of life (QoL-RLS, IRLS subscale "symptom impact") and in mental health status (SCL90-R). Sleep quality had improved at the end of the therapy (RLS-6 scale "sleep satisfaction"). Besides the global score of the SCL-90-R, the corresponding subscale "anxiety" had decreased significantly between baseline and the end of the course (from 6.0 (5.7) to 5.3 (4.7) points; $p=0.036$ ). These effects remained stable until the follow-up visit. In addition, improvements were noted in the WBS-5 and the SF-36 mental component summary

Table 1 Psychometric assessments of the group therapy: quality of life and psychopathological measures

\begin{tabular}{|c|c|c|c|c|c|}
\hline Scale & $\begin{array}{l}\text { Baseline } \\
\text { (BL) }\end{array}$ & $\begin{array}{l}\text { After } \\
\text { therapy } \\
\text { (END) }\end{array}$ & $\begin{array}{l}\text { p Value } \\
\text { (BL vs END) }\end{array}$ & $\begin{array}{l}\text { Follow-up } \\
\text { (FU) }\end{array}$ & $\begin{array}{l}\text { p Value } \\
\text { (BL vs FU) }\end{array}$ \\
\hline OoL-RLS ${ }^{12}$ & $28.6(12.8)$ & $23.4(13.1)$ & $<0.001$ & $21.2(9.7)$ & 0.001 \\
\hline SCL-90-R ${ }^{14}$ & $51.3(37.0)$ & $45.9(32.9)$ & 0.031 & $43.7(34.8)$ & 0.001 \\
\hline WBS- $5^{15}$ & $12.7(4.6)$ & $13.6(4.3)$ & 0.207 & $14.8(4.2)$ & 0.034 \\
\hline $\begin{array}{l}\text { SF-36: mental component } \\
\text { summary }\end{array}$ & $46.0(11.7)$ & $47.4(10.9)$ & 0.445 & $49.8(7.9)$ & 0.026 \\
\hline $\begin{array}{l}\text { SF-36: physical component } \\
\text { summary }^{13}\end{array}$ & $45.4(9.3)$ & $45.7(10.4)$ & 0.978 & $44.8(9.6)$ & 0.854 \\
\hline IRLS total score ${ }^{16}$ & $25.9(6.9)$ & $19.1(6.3)$ & $<0.001$ & $18.9(7.0)$ & $<0.001$ \\
\hline $\begin{array}{l}\text { IRLS subscale: impact of RLS on } \\
\text { daily life }{ }^{16}\end{array}$ & $6.0(2.3)$ & $4.2(1.9)$ & $<0.001$ & $4.0(1.7)$ & $<0.001$ \\
\hline $\begin{array}{l}\text { IRLS subscale: severity of RLS } \\
\text { symptoms }{ }^{16}\end{array}$ & $15.1(3.4)$ & $11.1(2.7)$ & $<0.001$ & $11.3(4.4)$ & $<0.001$ \\
\hline RLS-6: severity at bedtime ${ }^{17}$ & $5.2(3.3)$ & $3.1(2.7)$ & 0.008 & $3.0(2.6)$ & 0.004 \\
\hline RLS-6: severity during the night ${ }^{17}$ & $4.4(3.5)$ & $3.4(2.7)$ & 0.096 & $3.7(2.9)$ & 0.339 \\
\hline $\begin{array}{l}\text { RLS-6: severity during the day when } \\
\text { at rest }{ }^{17}\end{array}$ & $2.7(2.5)$ & $1.7(1.6)$ & 0.010 & $1.8(2.0)$ & 0.077 \\
\hline RLS-6: satisfaction with sleep ${ }^{17}$ & $6.4(2.4)$ & $4.5(2.6)$ & 0.009 & $5.0(2.2)$ & 0.035 \\
\hline
\end{tabular}

Values are given as mean (SD). Results of the mid-treatment evaluation are reported in the text.

BL, baseline evaluation; END, evaluation at the end; FU, evaluation 3 months after the end of therapy; 0oL-RLS, Quality of life in RLS ( $0=$ excellent, 54 = very bad. Note: item 8, asking for side effects of medication, was omitted); SCL-90-R, Symptom Check List-90 Revised Version (population-based reference value: 50 (10)); WBS-5, WHO-5 Well-Being Scale $(0=$ very bad,

25 = excellent; scores $\leqslant 13$ indicative of depression); IRLS, International RLS Severity Scale $(0=$ no symptoms, $40=$ very severe symptoms); IRLS impact subscale, sum score of the IRLS items 5, 9 and $10(0=$ no impairment, $12=$ very much impaired); IRLS severity subscale, sum score of the IRLS items $1,2,4,6,7$ and 8 ( $0=$ no symptoms, $24=$ very severe symptoms); RLS- 6 scales, for each item: $0=$ no symptoms, $10=$ very severe symptoms. 
score. At the mid-treatment evaluation, improvements in the IRLS global scale, the IRLS subscales and the RLS-6 (severity at bedtime) were already present (data not shown). The anxiety and the depression subscores of the Hospital Anxiety and Depression Scale ${ }^{18}$ and the total score of the Beck Depression Inventory ${ }^{19}$ did not change during the therapy. Unmedicated patients showed a tendency to benefit more than medicated patients, although there was no statistically significant difference between the groups.

\section{RLS severity scales}

Subjective ratings of RLS severity in the IRLS total score and the IRLS subscale "symptom severity" showed an overall improvement in RLS symptoms (see also table 1). The profiling of symptoms at different time periods during the day showed favourable changes in the RLS-6 scales "severity at bedtime" and "severity during the day when at rest", but no changes in the scale "severity during the night".

\section{Global rating of patients}

In the last session, patients were requested to rate different aspects of the coping therapy. The first part of this questionnaire comprised items about the global effect of the therapy. Patients rated the group therapy on a scale from 4 to 0 ("I agree fully" to "I do not agree") as specific for RLS-related issues (3.9 (0.3)), of feeling better educated about RLS (3.8 (0.4)), and feeling better able to cope with RLS $(3.2(0.8))$. In the second part, patients were asked to rank the most helpful elements of the therapy. Patients ranked as most helpful (in descending order) the mindfulness-based exercises (including breathing exercises), stress reduction strategies, diary-based analysis of factors aggravating RLS, and medical education.

\section{DISCUSSION}

We here present the evaluation of the first psychologically oriented approach to RLS management, the RELEGS therapy programme. The evaluations indicate disorder-specific effects on RLS-related quality of life and demonstrate the feasibility and high patient acceptance of the therapy programme.

An unexpected effect of the therapy was the significant improvement in RLS severity. The RLS-6 scales show that relief from symptoms occurred primarily when patients were awake but not during the night, indicating that the patients used the acquired coping strategies in their daily life. Furthermore, a change in the cognitive interpretation of RLS symptoms and its consequences during the course of therapy had possibly occurred-that is, a change in the subjectively perceived "bothersomeness" of the disorder.

We developed the group therapy both as a stand-alone treatment for unmedicated patients with RLS who do not need pharmacotherapy and as an adjunctive therapy for patients with partial remission of RLS symptoms under medication. We found no differences in the outcome measures between the two subgroups. The coping therapy therefore can be applied in both medicated and unmedicated patients. As our main concern was to evaluate the feasibility of the therapy programme, we did not have a control group and for this reason cannot exclude placebo effects. To confirm the efficacy of the coping therapy, randomised controlled studies (eg, using non-disorder-specific behavioural therapy and a standardised manual) are necessary and are currently being designed by our group.

A better understanding of the bio-psycho-social consequences of chronic diseases over the last few years has resulted in recognition of the importance of patient self-management and health literacy. ${ }^{20}$ Increasing evidence shows that adequate coping strategies-that is, adequate self-managementimproves outcome and reduces overall managed-care costs in chronically ill patients. ${ }^{21}$ Current treatment trials in RLS still focus on drug therapy, and comprehensive psycho-biological management strategies have yet to be developed for this patient population. The therapy presented in this paper may be an important step in the development of an integrated treatment approach to RLS management.

Acknowledgements: We thank the German RLS Patient Support Group, RLS e.V., Munich, Germany for an unrestricted grant for this study.

Funding: Unrestricted grant from the German RLS Patient Support Group, RLS e.V., Munich, Germany.

\section{Competing interests: None.}

Ethics approval: Ethics approval was obtained.

Patient consent: Informed consent was obtained for publication of the case details described in this report.

\section{REFERENCES}

1. Allen RP, Walters AS, Montplaisir J, et al. Restless legs syndrome prevalence and impact: REST general population study. Arch Intern Med 2005;165:1286-92.

2. Allen RP, Picchietti D, Hening WA, et al. Restless legs syndrome: diagnostic criteria, special considerations and epidemiology. A report from the restless legs syndrome diagnosis and epidemiology workshop at the National Institute of Health. Sleep Med 2003:4:101-19.

3. Vignatelli L, Billiard M, Clarenbach $P$, et al. EFNS Task Force. EFNS guidelines on management of restless legs syndrome and periodic limb movement disorder in sleep. Eur J Neurol 2006;13:1049-65.

4. Paulus W, Trenkwalder C. Less is more: pathophysiology of dopaminergic-therapyrelated augmentation in restless legs syndrome. Lancet Neurol 2006;5:878-86.

5. Abetz L, Allen R, Follet A, et al. Evaluating the quality of life of patients with restless legs syndrome. Clin Ther 2004;26:925-35.

6. Hornyak M, Kopasz M, Berger M, et al. Impact of sleep-related complaints on depressive symptoms in patients with restless legs syndrome. J Clin Psychiatry 2005;66:1139-1145.

7. Picchietti D, Winkelmann JD. Restless legs syndrome, periodic limb movements in sleep, and depression. Sleep 2005;28:891-8.

8. Winkelmann J, Prager M, Lieb R, et al. "Anxietas tibiarum" depression and anxiety disorders in patients with restless legs syndrome. J Neurol 2005;252:67-71.

9. Melbourne Academic Mindfulness Interest Group. Mindfulness-based psychotherapies: a review of conceptual foundations, empirical evidence and practical considerations. Aust N Z J Psychiatry 2006;40:285-94.

10. McCracken LM, Turk DC. Behavioral and cognitive-behavioral treatment for chronic pain: outcome, predictors of outcome, and treatment process. Spine 2002;27:256473.

11. Wittchen H-U, Zaudig M, Fydrich T. SKID. Srukturiertes Klinisches Interview für DSM-IV SCID (Structured Clinical Interview for DSM-IV). Göttingen: Hogrefe Verlag für Psychologie, 1997.

12. Kohnen R, Benes H, Heinrich CR, et al. Development of the disease-specific Restless Legs Syndrome Quality of Life (RLS-OoL) questionnaire. Mov Disord 2002;17(Suppl 5):P743.

13. Ware JE Jr, Sherbourne CD. The MOS 36-item short-form health survey (SF-36). I. Conceptual framework and item selection. Med Care 1992;30:473-83.

14. Derogatis LR. Symptom Checklist-90-Revised. In: Handbook of psychiatric measures. Arlington, VA: American Psychiatric Association, 2000:81-4.

15. World Health Organization. World Health Organization info package: mastering depression in primary care. Frederiksborg: World Health Organization, Regional Office for Europe, Psychiatric Research Unit, 1998.

16. The International Restless Legs Syndrome Study Group. Validation of the International Restless Legs Syndrome Study Group rating scale for restless legs. Sleep Med 2003;4:121-32.

17. Kohnen R, Oertel WH, Stiasny-Kolster K, et al. Severity rating of restless legs syndrome: validation of the RLS-6 scales. Sleep 2004;27(Abstract Suppl):A304.

18. Zigmond AS, Snaith RP. The Hospital Anxiety and Depression Scale. Acta Psychiatr Scand 1983:67:361-70.

19. Beck AT, Ward $\mathrm{CH}$, Mendelson $\mathrm{M}$, et al. An inventory for measuring depression. Arch Gen Psychiatry 1961;4:561-71.

20. Coleman MT, Newton KS. Supporting self-management in patients with chronic illness. Am Fam Physician 2005;72:1503-10.

21. Bodenheimer T, Wagner EH, Grumbach K. Improving primary care for patients with chronic illness. JAMA 2002;288:1775-9. 